\title{
IMPROVED WAVELET COMPRESSION ALGORITHM FOR COLOR IMAGE
}

\author{
S. Anantha Babu1, P. Eswaran ${ }^{2}$ and C. Senthil Kumar ${ }^{3}$ \\ ${ }^{1}$ Department of Computer Science and Engineering, V.V. College of Engineering, India \\ ${ }^{2}$ Department of Computer Science and Engineering, Alagappa University, India \\ ${ }^{3}$ Department of Computer Science, Erode Arts and Science College, India
}

\begin{abstract}
An image compression technique has been done in research in the recent years due to its clarity and quality compared to other techniques. Image compression based on wavelet is very important-role and occupies many applications. The objective of image compression is to help in storing the transmitted date in an efficient way by decreasing its redundancy. The wavelet compression reduces the size of the image data while retaining information and maintaining a certain wavelet compression. The proposed method of Improved Wavelet Compression (IWC) is presented in this paper. The proposed IWC gets a color image from the database. After receiving image, waveletTransformation using filter bank techniques are applied to the test image. After compression, the inverse IWC decompression algorithm receives compressed image and applied decompression technique. The image is generated and image quality is reconstructed and the original image is evaluated. The numerical measure parameters such as MSE, PSNR, are used to compare various images. From the experimental result, it is observed that the proposed method IWC gives a better compression ratio in 64.56 while compared to the existing methods.
\end{abstract}

Keywords:

RGB Space, Isolated Color Components, DCT, DWT, Improved Wavelet Compression (IWC), MSE, PSNR

\section{INTRODUCTION}

Data compression involves the development of a compact representation of information. Most representations of the information contain large amounts of redundancy. Redundancy can exist in various forms it may exist in the form of correlation: spatially close pixels in an image are collectively besides close in value. The redundancy might be due to context: the number of possibilities for a particular letter on a piece of English text is drastically reduced if the previous letter is a $q$. It can be probabilistic in presence: the letter e is much more likely to occur in a piece of English text than the letter $q$ [1].

The image is clearly a kind of redundant data, i.e. it contains the same information from a certain perspective of view. By performing data compression techniques, it is desirable to remove some of the redundant information contained in images. Image compression decreases the size in bytes of a graphics file without degrading the quality of the image to an unacceptable level [2]. The term includes many different types of repeated patterns. A wavelet-based compression scheme will try to approximate the data by adding together a number of basic patterns known as wavelets that are weighted by the specific coefficients. If the program does a relevant task, then the sum is pretty close if not exactly the same as the original. Then the coefficient scan proceeds the position of authentic and save plenty of space [3].

Transform coding at low rates tends to give the reconstructed image a blocky appearance. This has led to the increasing popularity of sub band and wavelet-based schemes. The implementation for both sub band and wavelet-based schemes are similar. The input is filtered through a bank of filters, called the analysis filter bank. The filters cover the entire frequency range of the signal. The capacity of specific filter is the exclusive fragment of the bandwidth of primary signal, the Nyquist principles illustrate that, the sum of samples prescribed through the amount of the filter remains a minimum number of illustrating per second needed at the prescription of the filter. The output of the filters is sub sampled or decimated and encoded. The sub sampled aggregate values are grouped, and the group of quantization order is encoded. At the decoder, after the received samples are decoded they are up sampled by the insertion of zeros between the received samples and filtered using a bank of reconstruction filters [4].

The blocking is the relatively constant structure of compression achieved, and numerous aspect its existence to the lack of the block discrete cosine transform to endure of correlated among blocks. Although there is a principle of the blocking form can further be defined by as long as the compression of a specific block, which is the path concerned the individual block of the image to which the DCT is correlated is accomplished, and the results are developed from DCT block. The DCT is one dimension, the signal to be remodeled to specify $z[n], 0 \leq n \leq N$, which is further to defined by the term of $\mathrm{N}$ column vector $\mathrm{z}$. The element of DCT matrix $H=\{H[k, n]\}$ are defined as [5],

$$
H=\left\{\frac{\frac{1}{\sqrt{N}}}{\frac{2}{\sqrt{N}} \cos \left[\frac{\pi(2 n+1) k}{2 N}\right]}\right\}
$$

where, $\mathrm{k}=0,0 \leq n<N, 1 \leq k<N, 0 \leq n<N$. The DCT is $y=H z$. After all, the DCT is a real entire transform, $H^{-1}=H$ and the inverse DCT is define by $z=H^{t} y$. Let $h_{k}^{t}$ signify the $k^{\text {th }}$ row of $H$.

$$
z=\sum_{k=0}^{N-1} h_{k} y[k]
$$

The Process of JPEG method follows the steps to construct the image is [6],

1. The given image is divided into $8 \times 8$ blocks of pixels.

2. It is operating from left to right and top to bottom of the DCT is coordinated to entire blocks.

3. In an individual block is constricting over quantization. 
4. The group of image is compressed the blocks that create the image is reserved in an excessively moderate volume of location.

5. The image is reconstructed around decompression; the process is handling the Inverse Discrete Cosine Transform (IDCT).

Hence, image organize the large part of transmission data, we focus on the work in computing efficient ways of image compression using Improved Wavelet Compression (IWC). We are therefore considering Filter Bank implementation using IWC compression and decompression algorithm.

This paper is organized as follows; in section 2 reveals the literature review of the DCT compression and wavelet methods. Section 3 presents the Discrete Cosine Transform and section 4 describes Discrete Wavelet Transform. In section 5 presents the principles of wavelet transform. Section 6 reveals that the proposed methodology of wavelet transformation and explains how fast wavelet transformation filter bank and proposed method implementations. Section 7 discusses the experimental results. The conclusion and future direction, are discussed in section 8 .

\section{LITERATURE REVIEW}

The digital color image can be examined as a three admired (channels) specific method $I=I(\mathrm{x}, \mathrm{y})$ represent onto a plane. It is algebraic representation is accessible through an $N$ by $M$ by 3 matrices $A$. Thus, each entry of $A$ is a three component integer vector (pixel color) expressing an intensity value at discrete locations $(x, y)$ with a precision $p$ (for instance, one bit for any channel). An each component or layer of the image can be viewed as a single channel image, which, under particular conditions. RGB is the most frequent storage format for images, other formats may be better for compression. The major process in lossy data compression in which data cannot be recovered completely is the quantization phase, which accomplishment a data reduction based on their low information content. This is not optimal for RGB images. After all, for three layers, this can lead to the destruction of some low coefficients in a channel in a convinced spatial location, although the identical coefficients in the other layers are not defeating because they carry high information content. When restore, the image at that location is a high visual distortion is received. The theory of analyzing the three layers independently is legitimate only if they are not correlated with respect to the visual appearance [6].

RGB is the essential and, most commonly used color space. It uses the proportions of red, green, and blue that are proportion to a minimum and maximum values for each component (for example, $0 \mathrm{x} 00$ through $0 \mathrm{xFF}$, or 0.0 through 1.0). Most colors in the detectable spectrum can be converted, after all not completely. This scheme is based on the additive properties of color. [6]. The three color spaces have unique $\mathrm{YCrCb}$ is the desired one benefit in the image processing because,

1. For using $\mathrm{YCrCb}$ is not similar in color region, therefore ever region can be evaluated distinctly.

2. Human eyes are higher observant to the innovation of highlight than of color, therefore $\mathrm{Cr}$ and $\mathrm{Cb}$ quantity can be rebate more actively than $\mathrm{Y}$ space to get good compression ratio.[7]
3. Downsampling/Upsampling: Data rebate of attainable constituent $\mathrm{Cb}$ and $\mathrm{Cr}$ is attained in JPEG compression as needed. Essentially, the JPEG compression software adapts the 4:1:1 sampling as the loss of data. The 4:1:1 sampling process of four data in the luminance component, $\mathrm{Y}$ and one data for every chrominance component $\mathrm{Cb}$ and Cr subsequently two horizontal pixels close upon two vertical pixels. The 4:4:4 sampling is still used, which clause no data minimization of chrominance components. Restoration of chrominance components $\mathrm{Cb}$ and $\mathrm{Cr}$ to full size is observed in JPEG decompression if required.

4. The Inverse DCT of the source image is reconstructed into the spatial frequency domain with DCT in JPEG compression. The compressed image information in the spatial frequency domain is converted into the image domain by reverse DCT among JPEG decompression.

5. Quantization/Dequantization: DCT-coefficient data are quantized in JPEG compression, the quantization table is demonstrated in this step to control the compression level of an image. In quantization level in the lossy process. Dequantization for JPEG decompression is complete by using the quantization table involved in the compressed image essential [8].

Many image applications share an important goal with image compression, i.e., to reduce the number of bits needed to represent the content of the original image. We now study the advantages and disadvantages of using the DCT to transform for image compression. The DCT is used in the JPEG (Joint Photographic Experts Group) compression encoding and decoding (codec) standard. In order to improve spatial localization, JPEG divides images into $8 \times 8$ non-overlapping pixel blocks and applies the DCT to each block. An exclusive quantizes of the 64 frequency components consistently applying a quantization step described by a table designed coordinate to the human visual system acuity to distinct repetition. A smaller quantization step, or equivalently higher quantization accuracy, is used to quantize a frequency component to which the HVS is more sensitive. [9] Haar Wavelet is an Orthonormal wavelet bases are an evolution of the Haar bases [10]. In 1909, Haar described orthonormal bases, defined on $[0,1]$, namely $h_{0}(x), h_{1}(x), \ldots h_{n}(x), \ldots$ other than the Fourier base such that for any continuous function $f(x)$ on $[0,1]$, the series is:

$$
\sum_{j=1}^{\infty}<f, h_{j}>h_{j}(x)
$$

converges to $f(x)$ uniformly on $[0 ; 1]$. Here, $\langle u, v\rangle$ denotes the inner product of $u$ and $v$ :

$$
<u, v>=\int_{0}^{1} u(x) \overline{v(x)} \cdot d x
$$

where $\bar{v}$ is the complex conjugate of $v$, which equals $v$ if the function is real-valued.

One version of Haar's construction $[10,11,12]$ is as follows:

$$
\begin{aligned}
& h(x)=\left\{\begin{array}{c}
1, x \in[0,0.5] \\
-1, x \in[0,0.5] \\
0, \text { elsewhere }
\end{array}\right. \\
& h_{n}(x)=2^{j / 2} h\left(2^{j} x-k\right)
\end{aligned}
$$


where, $n=2^{j}+k, k \in\left[0,2^{j}\right], x \in\left[k 2^{-j},(k+1) 2^{-j}\right]$

There are certain constraints in using Haar's construction. Because Haar's base functions are discontinuous step functions, they are not significant for determining the smooth functions with continuous derivatives. Since images are often contains smooth regions, the Haar wavelet transform does not maintain satisfactory results in many applications.

The comprehension for implementing wavelet transform based compression, JPEG compression ratio based on DCT (Discrete Cosine Transform) retain several improvements like as accuracy, appropriate action, and availability of special purpose hardware for implementation. Finally, the input image is discontinued; correlation beyond the block barrier cannot be rejected. This result is observable and troublesome "blocking artifacts" especially at minimum bit rates. The wavelet transform has been mostly used in signal processing research, exactly in image compression. In certain usage, wavelet-based method can retrieve the enhanced attainment than other coding methods like the DCT based method. After all, there is no need to block of the original image and its basis method have a variable length, wavelet based coding methods can abstain blocking artifacts. The wavelet coding also inclusion to the extended communication of images [13]. Uncertainly the researchers target on Discrete Wavelet Compression Technique. In this paper, the literature review is deliberate completely the recent direction. Therefore, it motivates and propose a remodeled access to the extant process of making conditions.

\section{DISCRETE COSINE TRANSFORM}

The DCT image compression, the original image is initially divided into a number of small blocks in the scheme of blocks with a normal block size of $8 \times 8$ pixels and classify of the separate image block is executed independently. In DCT, the principle of no loss of the original image information as it completely remodels the image into a domain that compressed in an effective way [12]. In DCT provides the respective of 64 DCT belonging of the continually quantized image. The Zig-zag scanning is enforced to rearrange the coefficients preceding to entropy coding. The minimum repetition of non-zero constituent are arranged at the inception of the bit stream succeed by the huge number of units [14]. In Fig.1, illustrates the mechanism of zigzag scanning. The discrete cosine component that consists of the source information around the image is summarized exclusively. Finally, the output of the drift subsequently the zig-zag examination is entropy embedding.

The DCT originates of image compression produces sufficient compression capability and it provides lesser memory usage after the entire compress is performed on limited specific of image blocks [14]. Despite, the tiling of the blocks motivates blocking artifacts downfall the performance, specifically at minimum bit rates [13] [15].
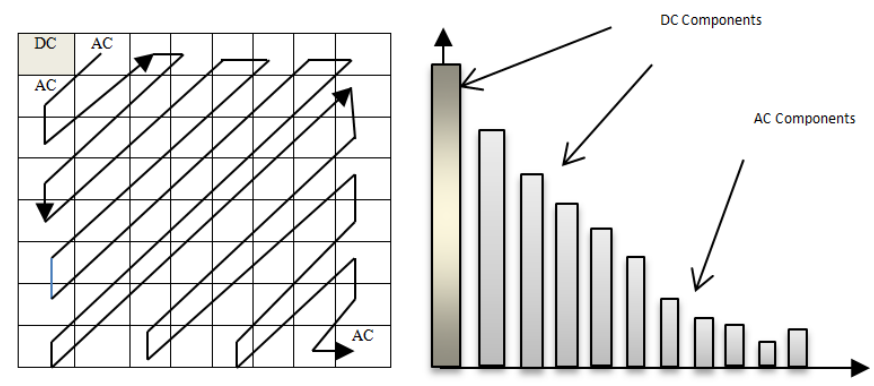

Fig.1. Zig- Zag Scanning

\section{DISCRETE WAVELET TRANSFORM}

The wavelets are signals which are mostly unreliable in model and characteristics in time and scale. A wavelet is a waveform that has a moderate value of zero and conveniently finite continuation. The DWT produce an image as a value of these wavelet methods, with a distinctive section and a range [16]. In DWT procedure the image input is split into a set of high pass coefficients (detail) and low pass coefficients (approximate) [17]. In DWT the original image is originally divided into a group of $32 \times 32$ blocks and then separate block has reached over the two filters: In the first level disintegration the prescription of the image is split into four multi resolutions, non-overlapping sub-bands LL, HH, LH, and HL that is also assigned as coefficients.

The sub band LL is stated as relating to the coefficient acquire the common value of DWT coordinate the sub bands of LH, HL, and $\mathrm{HH}$ are further exclusive as different coordinates which presents as the great system of discrete wavelet coefficients. The LL coefficients are convert into the next level by concede all the identical coordinates. To achieve the appropriate compression ratio the LL coordinates are reach over a dependable scaling aspect.

In case of the discrete wavelet image is not intense into non overlapping two dimension blocks and as the revolution is tested to the whole original image, it avoids blocking invention and consequence in a superior to compression ratio as correlated to discrete cosine transform [18].

\section{PRINCIPLES OF WAVELET TRANSFORM}

The image wavelet transform retain the rapid algorithm of two dimensional wavelet transform. The source image is crumbled into four sub-bands consequently fast high-pass filter and lowpass filter [19].

The four sub-bands are LL, HL, LH and HH eventually. The LL is a slightest repetition sub-band of the interchangeable image. The HL is a highest reiteration sub-band of the horizontal representation of the image. The $\mathrm{LH}$ is a better frequency subband of the vertical characteristics of the image. $\mathrm{HH}$ is a high recurrence sub-band of the diagonal depiction of the image.

This technique is assigned the first level of wavelet decomposition. The minimum reiteration sub-band can be subsequently decayed into four sub-bands. The decomposition can be incredibly renovated in theory. But the people must abide the element of the reconstructed image into commitment. Hence, the people refuse of disintegrate image as well as the fifth intend. The researchers are routinely accepted the third level. 
The wavelet decomposition is shown in Fig.2 of third attempt. The digital image data aren't scaling down and cannot recognize image information compression succeeding the wavelet transform. In spite of the initiative of the entire digital image is reconstructed ensuing this transform. The image of minimum reiteration sub-band respond of considerable data.

The values of high frequency sub-band, be adjacent to zero, the higher the frequency the more exempt this situation. The partial image is the lesser frequency is essential part of which can complete the image subdivision.

So the researchers yield more recognition of the discriminating later wavelet transforms and involve appropriate method to measure the image coordinates for achieve adequate compression.

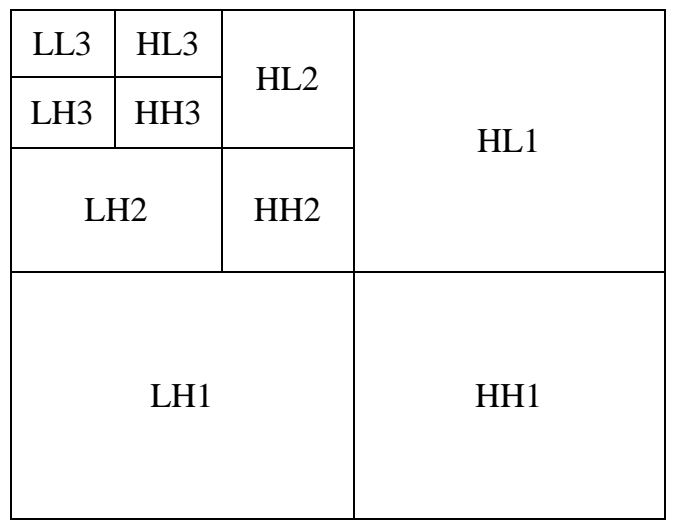

Fig.2. Three Level Wavelet Transformations

\section{PROPOSED METHODOLOGY}

\subsection{WAVELET TRANSFORMATION USES FILTER BANK}

The main objective of compression is explicit an initial set of data, using some smaller set of data, either with or without loss of information. The Fourier transform is the heart of many image processing applications, even though the Fourier transform works well with periodic signals it is not well affected with signals having sharp peaks and valleys. In addition, one of the major advantages this advantage of the Fourier transform is the loss of timing information of the signals. To overcome these issues, this paper presents 1D simplest Haar-wavelet transforms.

Let us consider 2D sub image data as below.

$$
\text { Sub Image }=\left[\begin{array}{l}
{\left[\begin{array}{ll}
9 & 7 \\
3 & 5
\end{array}\right]\left[\begin{array}{ll}
4 & 2 \\
3 & 2
\end{array}\right]\left[\begin{array}{ll}
5 & 4 \\
2 & 2
\end{array}\right]\left[\begin{array}{ll}
5 & 5 \\
8 & 9
\end{array}\right]} \\
{\left[\begin{array}{ll}
3 & 3 \\
2 & 4
\end{array}\right]\left[\begin{array}{ll}
4 & 4 \\
6 & 7
\end{array}\right]\left[\begin{array}{ll}
8 & 7 \\
2 & 4
\end{array}\right]\left[\begin{array}{ll}
6 & 5 \\
4 & 3
\end{array}\right]} \\
{\left[\begin{array}{ll}
2 & 3 \\
5 & 6
\end{array}\right]\left[\begin{array}{ll}
7 & 8 \\
3 & 5
\end{array}\right]\left[\begin{array}{ll}
5 & 4 \\
8 & 9
\end{array}\right]\left[\begin{array}{ll}
6 & 7 \\
8 & 5
\end{array}\right]} \\
{\left[\begin{array}{ll}
2 & 4 \\
5 & 6
\end{array}\right]\left[\begin{array}{ll}
7 & 9 \\
8 & 6
\end{array}\right]\left[\begin{array}{ll}
3 & 5 \\
6 & 7
\end{array}\right]\left[\begin{array}{ll}
8 & 9 \\
3 & 5
\end{array}\right]}
\end{array}\right]
$$

With 4 [64] sub block pixels are having the following gray scale values and can be represented in the Haar basics in 1D as [ 9 73 5]. After arranging the linear 1D elements, average pixels are found out pairwise, together, to get the new lower resolution pixels are indicated as follows [8 4]. In this process, we have lost some information. To restore that information, it needs to store some other reconstructed parameters. In the above example, we will select 1 for the first detail co-efficient; because, the average is 1 less than 9 and 1 more than 7. This single number allows as recovering the first 2 pixels of the original image. In a similar manner, the second detail co-efficient is -1 , because $4+(-1)=3$ and $4-(-1)=5$. Finally, the Improved proposed Haar method have decomposed the original image in a lower resolution version and detail co-efficient as follows, reproducing this mechanism repeatedly gives the full decomposition.

\begin{tabular}{|c|c|c|}
\hline Resolution & Average & Detail co-efficient \\
\hline 4 & {$[9735]$} & - \\
\hline 2 & {$[8,4]$} & {$[1-1]$} \\
\hline 1 & {$[6]$} & {$[2]$} \\
\hline
\end{tabular}

Thus, for the 1D Haar basis, the wavelet transform sub image for 4 pixel image is given by the matrix. [6 $\left.\begin{array}{llll}6 & 2 & 1 & -1\end{array}\right]$. The way, we computed wavelet transform, by recursively averaging and differencing co-efficient are called filter banks. In this process, there is no loss or gain of information. The original and transformed image, both have the 4 co-efficient. Given the transform, we can reconstruct the image of any resolution by recursively adding and subtracting the detail co-efficient from the lower decision adaption as shown in Fig.3.
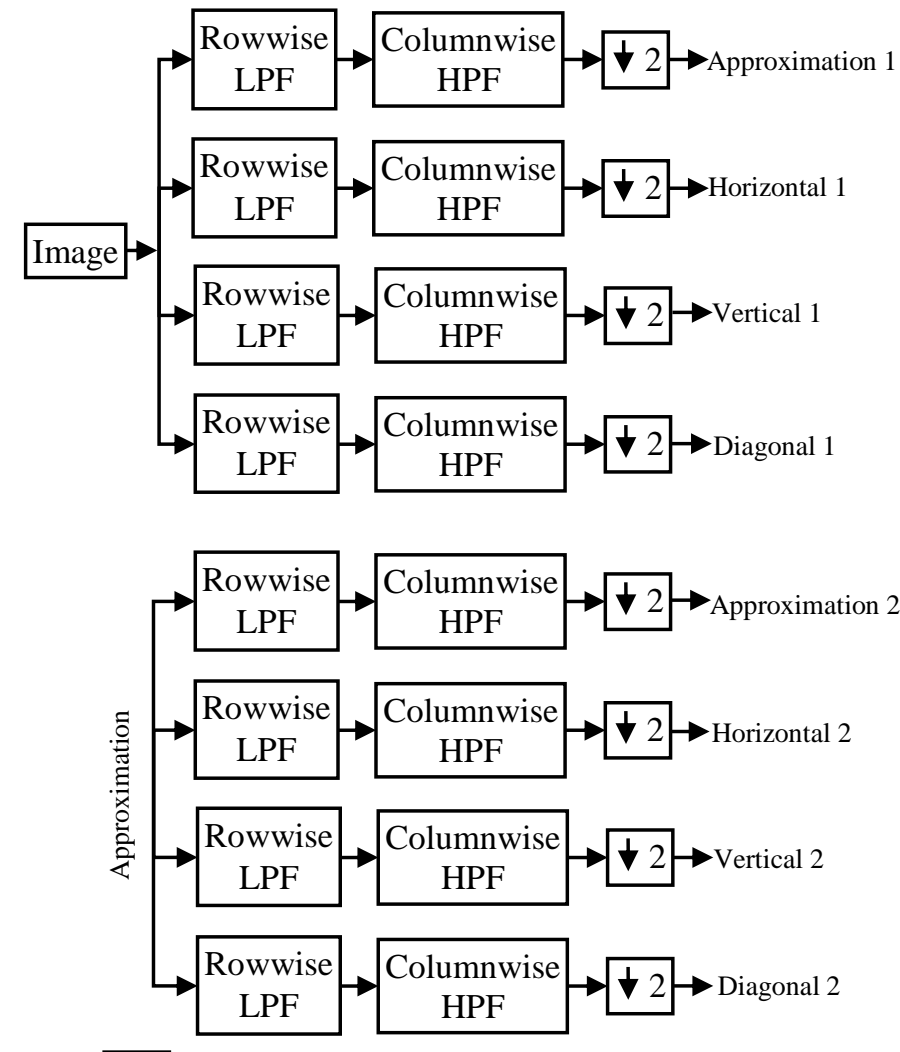

$\downarrow 2$ Downsample by ' 2 ' rowwise and columnwise

Fig.3. Filter Bank Down Sampling

Approximation 2 is further decomposed to get a further level approximation and detail (horizontal, vertical and diagonal) coefficient. To recover the original image from approximation 2, 
horizontal 2, vertical 2, diagonal 2, horizontal 1, vertical 1, diagonal 1 matrix as presented below in Fig.4.

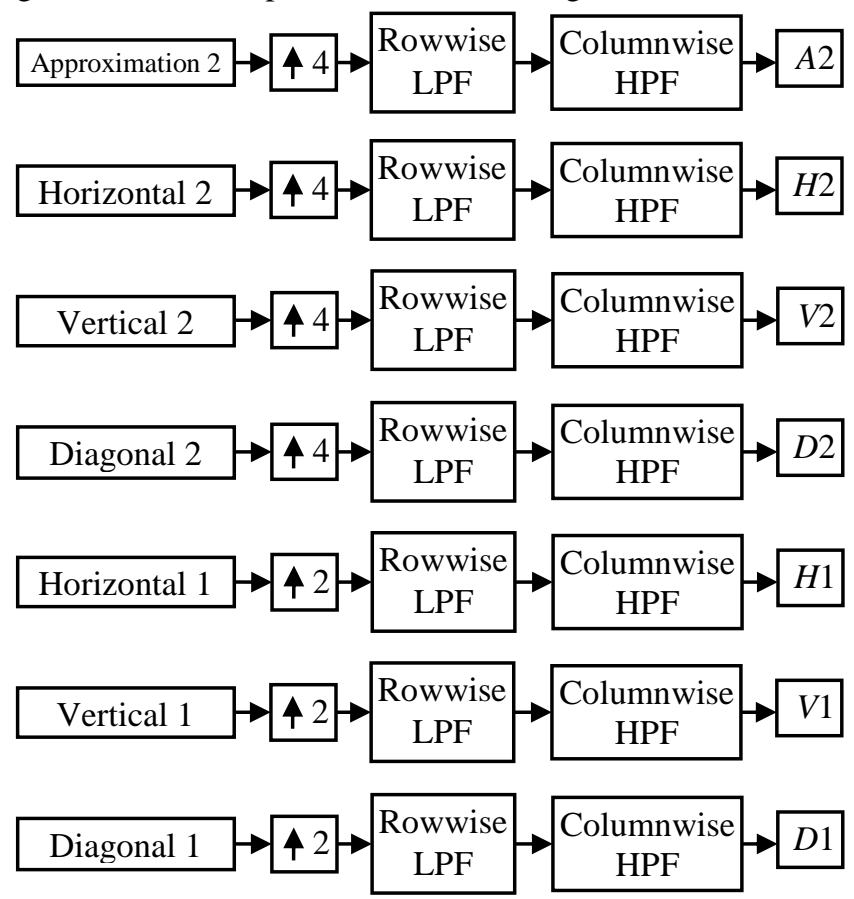

$\downarrow 2$ Upsample by '2' rowwise and columnwise

$\downarrow 4$ Upsample by '4' rowwise and columnwise

Fig.4. Filter Bank Up Sampling

Reconstructed Image $=A 2+H 2+V 2+D 2+H 1+V 1+D 1$

The filters are effete for restoration are entitled reconstruction filters. This filter used for decomposition is popular decomposition filters. There are four filters employing in filter bank positioned discrete wavelet transform.

1. Low pass decomposition filter

2. High pass decomposition filter

3. Low pass reconstruction filter

4. High pass reconstruction filter

It confides in the type of filters like (i) Daubechies, ' $\mathrm{db} 1$ '... 'db45', (ii) coif lets, 'coif' ... 'coif5'.., the wavelet transform is called as Daubechies WT, Coiflets WT, etc. [20].

The performance of wavelet transforms established on compression is feasible by applying a set of finite impulsive response filters in the way that low pass and a high pass filter. The concept of 1D wavelets can further be continued for 2D wavelets. In two dimensions, eventually one level of decomposition, there will be four frequency bands LL, HL, and HH, LH, HL and HH are often inadequate as a about to the images are low-frequency adapts. The second level of decomposition frequently concern the exclusive LL band. Most of all, there will be $(3 N+1)$ distinct frequency bands contain the $3 \mathrm{~N}$ high frequency bands and specific LL frequency band. 2D DWT presented in Fig.5. Equivalent to the aforementioned split in Fig.2, DWT is consuming the original image into various sub images.

\begin{tabular}{|c|c|c|c|c|}
\hline $\mathrm{LL}^{4}$ & $\mathrm{LH}^{4}$ & \multirow{2}{*}{$\mathrm{LH}^{3}$} & $\mathrm{LH}^{2}$ & \multirow{2}{*}{$\mathrm{LH}^{1}$} \\
\cline { 1 - 2 } $\mathrm{HL}^{4}$ & $\mathrm{HH}^{3}$ & & \\
\hline \multicolumn{3}{|c|}{$\mathrm{HL}^{2}$} & $\mathrm{HH}^{2}$ & \\
\hline \multicolumn{3}{|c|}{$\mathrm{HL}^{1}$} & $\mathrm{HH}^{1}$ \\
\hline
\end{tabular}

Fig.5. Four Level Wavelet Transformations

The IWC method applies the filter bank technique to find best computation time and compression ratio. Let $\mathrm{M}$ and $\mathrm{N}$ denotes number of rows and column of the image using filter bank, therefore the number of multiply operations can be reduced about $4\left(1-1 / 4^{L}\right) \times M \times N$ all over the whole image compression process which analysis part of $L$ level decomposition and $L$ level reconstruction. Therefore the wavelet can reduce the computation time and improve the compression ratio. The maximum level of decomposition can be determined using the formula,

$$
\text { Maximum level of decomposition }=\log _{2} X_{\text {max }}
$$

where, $\log _{2} X_{\max }$ is the maximum size of a given image.

\subsection{ALGORITHM}

\section{Compression}

Step 1: Get a gray or color image with different format.

Step 2: Arrange reshape size with $512 \times 512$ images.

Step 3: An image is divided into a number of small size nonoverlapping blocks.

Step 4: Convert block elements into Sequential order.

Step 5: Apply IWC to block elements using Filter Bank.

Step 6: Find Average Pairwise for Block

$$
\{\operatorname{elem}(0)+\operatorname{elem}(1)\}=\left[\frac{\text { Sum }}{2}\right]
$$

Step 7: Arrange Blocks elements into Sequence Order.

Step 8: Repeat this process until last block.

Step 9: Generate Compression ratio for all Levels.

Step 10: Stop.

\section{De-Compression}

Step 1: Read the compressed image.

Step 2: Convert the compressed image into matrix format.

Step 3: Divide into a number of non-overlapping blocks

Step 4: Arrange the gray / color scale values in 1D Sequence order.

Step 5: Apply Inverse IWC to get the original value

$$
\mid \text { elem }(0)-\text { Average } \mid=\text { Average }+1 \Rightarrow \text { Original Value }
$$

Step 6: Get the reconstructed image.

Step 7: Repeat step 2 to step 6 to process the whole sub block of compressed image. 
Step 8: Concatenate all reconstructed image to get back original image.

Step 9: Stop.

\subsection{PROPOSED IWC BLOCK DIAGRAM}

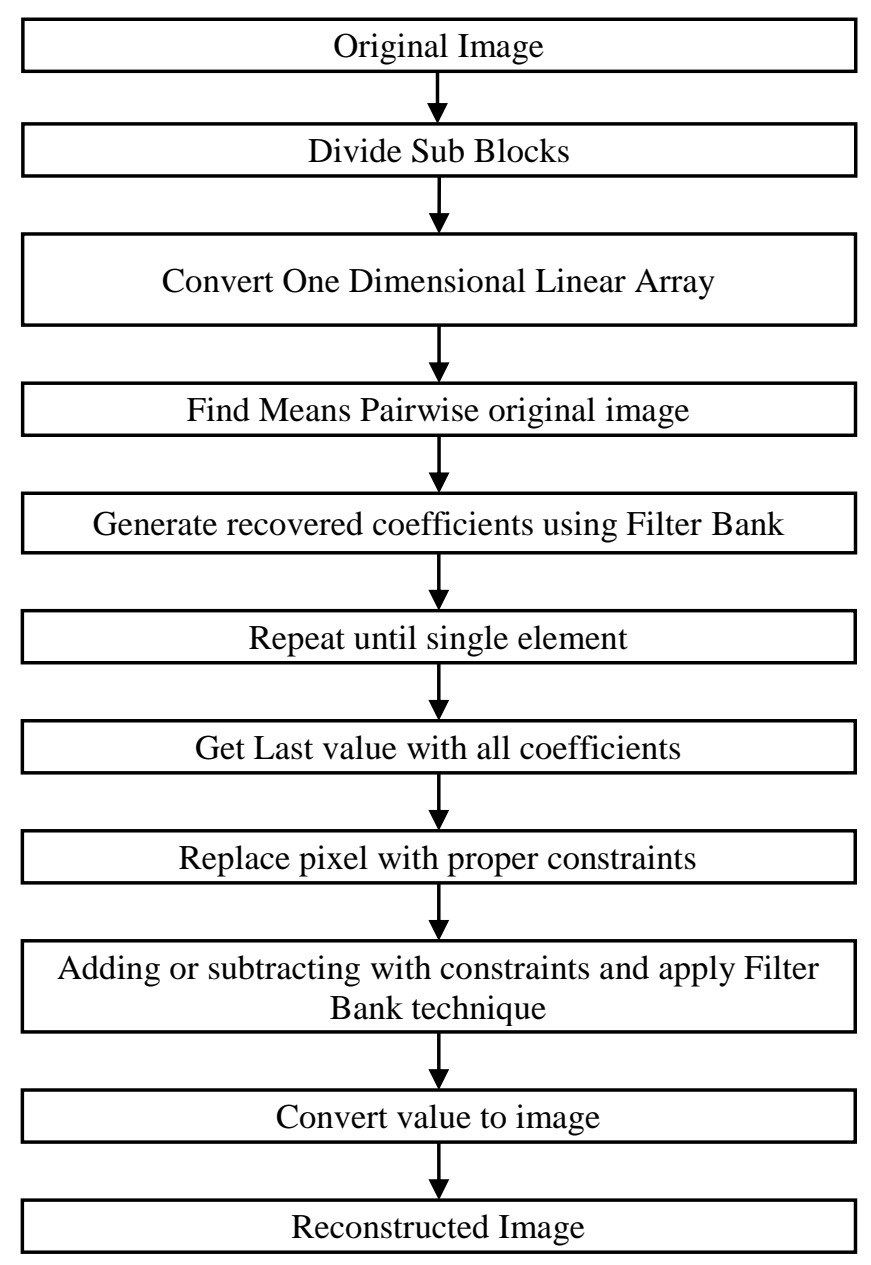

Fig.6. IWC Compression and Decompression

\section{RESULTS AND DISCUSSION}

\subsection{EXPERIMENTAL RESULTS}

In this section, the approximate decision of the parameter is normally used for image compression analysis has been explored. For the objective evaluation MSE, RMSE PSNR, CR, BR and CT have been discussed in the next section. The lower value of MSE indicates better picture quality. There is an inverse relationship between MSE and PSNR. Hence, the larger PSNR value gives the better image quality. Compression ratio indicates the efficiency of compression technique, more the compression ratio, less memory space required. Hence, the more compression ratio is always desirable without the trade off in image quality.

The Proposed IWC algorithms were implemented in MATLAB Version 13, a simulation tool. The evaluation parameters (PSNR, CR, MSE and BR), sub-sampling, quantization and scaling routines were calculated in MATLAB. The proposed algorithm is better compression ratio and Computation Time. Here, the IWC method performing up to four

levels of decomposition to obtain the coefficient matrix which is very much necessary for the compression purpose. In this work carrying out four level decomposition, to achieve 12 sub band images, which receive the Approximation information for Horizontal details, Vertical details and Diagonal details. After decomposition of the image, this proposed research method obtaining the coefficient matrix.

The IWC algorithm has been applied on more than 200 test images but for this method only three various test images have been provided. The images were obtained from USC-SIPI image database [23]. All images are RGB color images and the size is $512 \times 512$. The original pepper image is shown in Fig.7(a). In Fig.7(b) shown is Filter Bank Level 1, Block Size is 28956 Compressed Size is 883 , The PSNR value is 22.16 is calculated. Similarly the Filter Bank Level has been increasing for reduced the Block Size. The Level 2 Sub Sampling is shown in Fig.7(c) likewise, this paper calculated PSNR, MSE and CR for all Sub Levels. The MSE value is gradually decreased for better quality.

The Fig.7(d) shows the decoded images, IWC using the DWT Filter Bank methods to reduce the Block Size for visually shows quality image. The Fig.7(e) is illustrating PSNR versus the compression ratio for Peppers image used in the IWC for Level 4 Sub band. The Fig.7 (f) Sub Band Level 5 shows the Compression Ratio 45.26. In Fig.7(g) Sub Band Level 6 indicates the MSE 549.88 is gradually decreased for good quality of decomposition. Also, the PSNR value is gradually increased. The Fig.7(h) shows the reconstructed images of Sub Band Level 7 image Bit Rate is noted 211.36. Consistently Sub Band Level 8 shown in Fig.7(i). From Fig.7(j) and Fig.7(k) indicated by Sub Band Level 9 and 10 respectively. It shows the PSNR value is 34.95 and 35.15 measured with increased compatibility. Next, the IWC method finding the reduced block size Sub Band level 9 and 10.

Finally, the Sub Band Level 11 and 12 shows in Fig.7(1) and Fig.7(m). The Final Sub Level is completely reduced and MSE value is 295.25 to fine tune, the quality of the given original Pepper image. The PSNR, MSE, CR, CT and BR parameter are shown in the Section B Table.1.

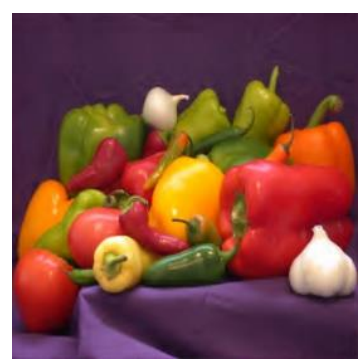

(a)

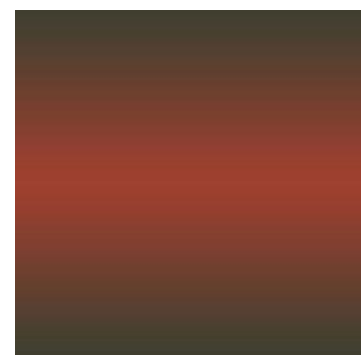

(c)

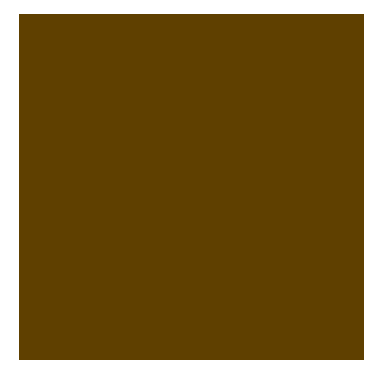

(b)

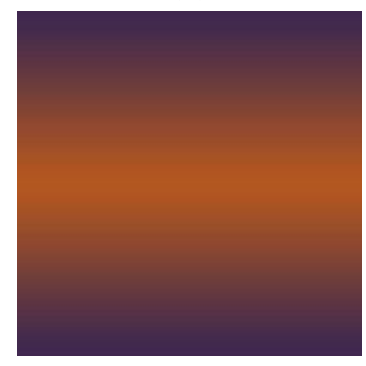

(d) 


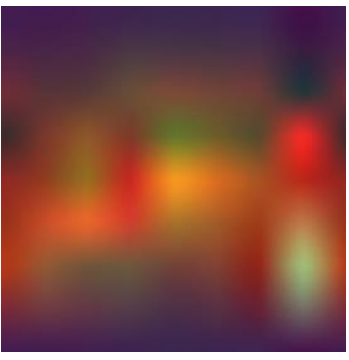

(e)

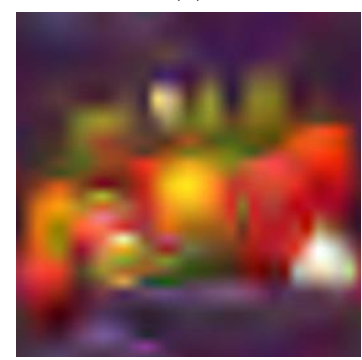

(g)

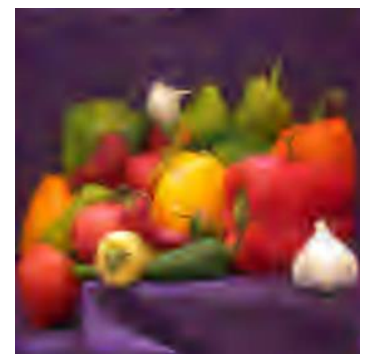

(i)

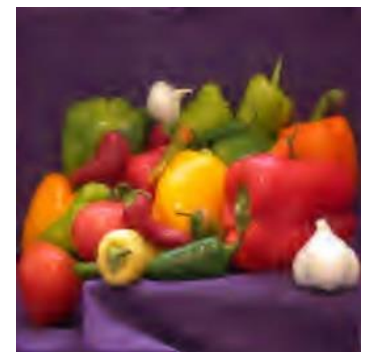

(k)

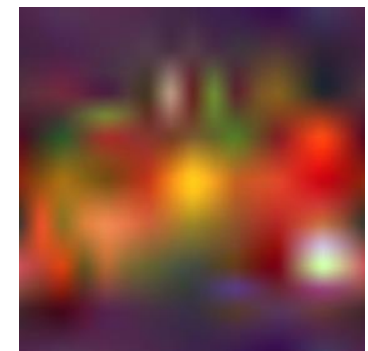

(f)

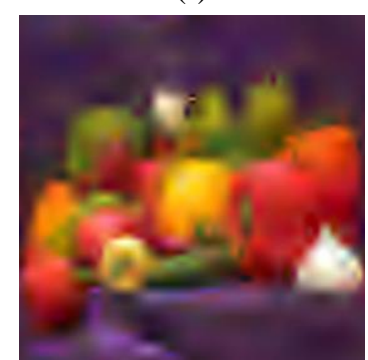

(h)

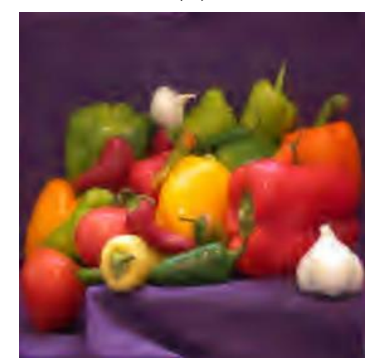

(j)

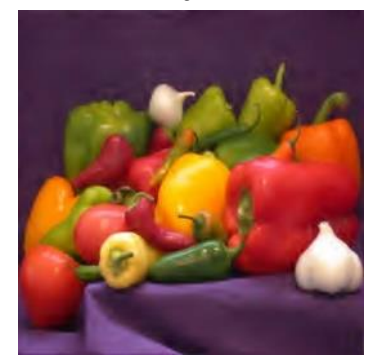

(1)

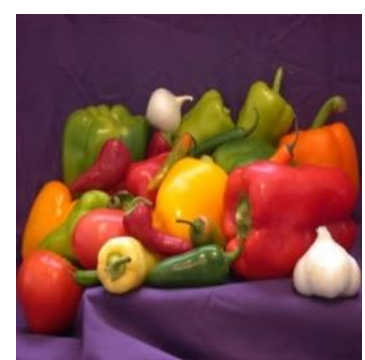

(m)

Fig.7. (a). Original Image (b). Level 1 (c). Level 2 (d). Level 3 (e). Level 4 (f). Level 5 (g). Level 6 (h). Level 7 (i) Level 8

(j) Level 9 (k) Level 10 (l). Level 11 (m) Level 12

The original Mandril image is shown in Fig.8(a). In Fig.8(b) shown is Filter Bank Level 1, Block Size is 78570 and Compressed Size is 883 , The PSNR value is 22.07 is calculated. Similarly the Filter Bank Level has been increasing for reduced the Block Size. The Level 2 Sub Sampling is shown in Fig.8(c).

The Fig.8(d) shows Sub Sampling Level 3, IWC uses the DWT Filter Bank methods to reduce the Block Size for visually shows quality image. The Fig.8(e) is illustrating the PSNR value 26.52 in Filter Bank Level 4. The Fig.8(f) Sub Band Level 5 shows the CR noted with 39.12. In Fig.8(g) Sub Band Level 6 indicates the MSE 1627 is gradually decreased for good quality of decomposition. Also, the PSNR value is gradually increased 30.06. The Fig.8(h) shows the reconstructed images of Sub Band Level 7 image Bit Rate is noted with 512.89. Consistently Sub Band Level 8 shown in Fig.8(i).

From the Fig.8(j) and Fig.8(k) indicated by Sub Band Level 9 and 10 respectively. It shows the PSNR value is 33.24 and 36.01 measured with increased compatibility. Next, the IWC methods finding the reduced block size Sub Band level 9 and 10.

Finally, the Sub Band Level 11 and 12 shows in Fig.8(1) and Fig.8(m). The Final Sub Level is completely reduced and MSE value is 271.13 to fine tune, the quality of the given original Mandril image. The PSNR, MSE, CR, CT and BR parameter are shown in the Section B Table.2.

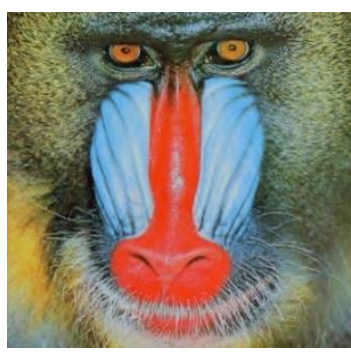

(a)

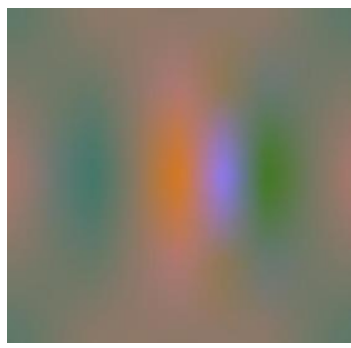

(c)

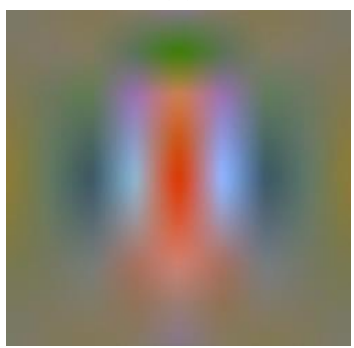

(e)

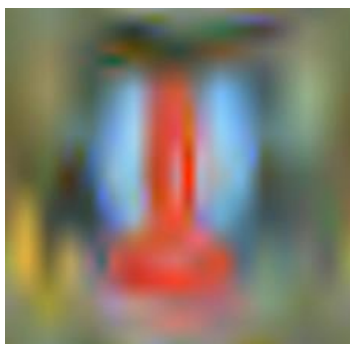

(g)

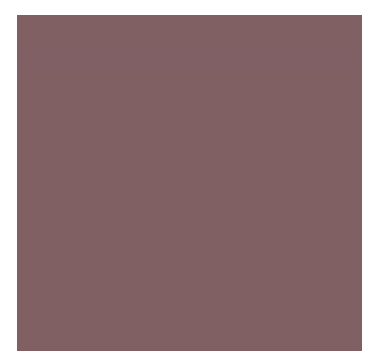

(b)

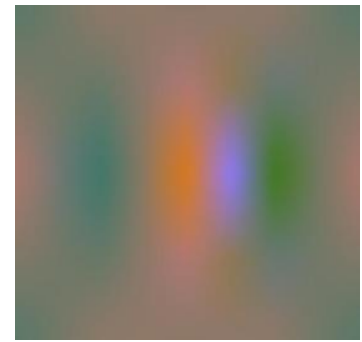

(d)

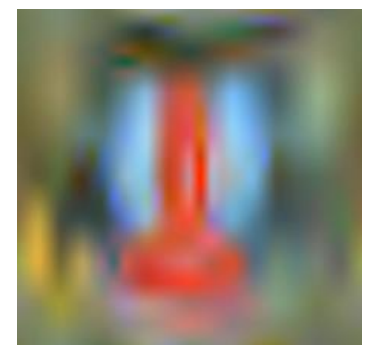

(f)

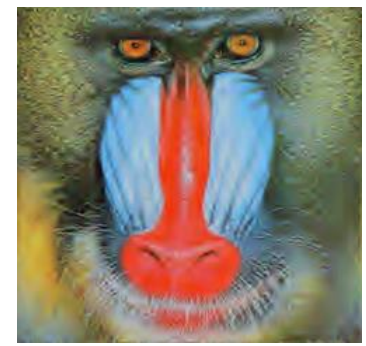

(h) 


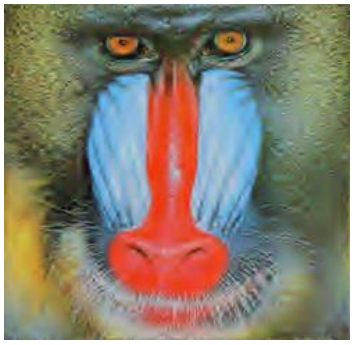

(i)

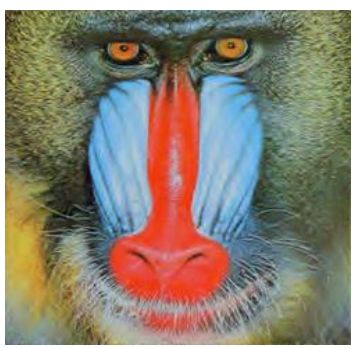

(k)

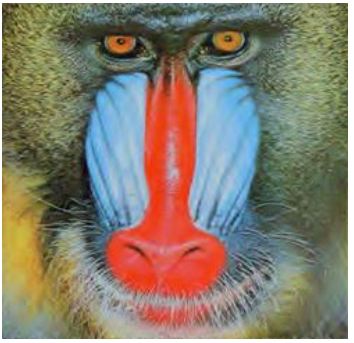

(j)

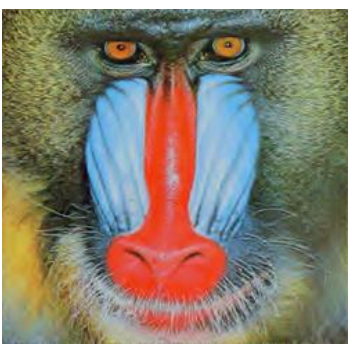

(1)

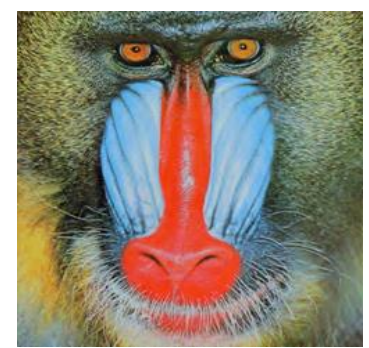

(m)
Fig.8. (a). Original Image (b). Level 1 (c). Level 2 (d). Level 3 (e). Level 4 (f). Level 5 (g). Level 6 (h). Level 7 (i) Level 8 (j) Level 9 (k) Level 10 (l). Level 11 (m) Level 12

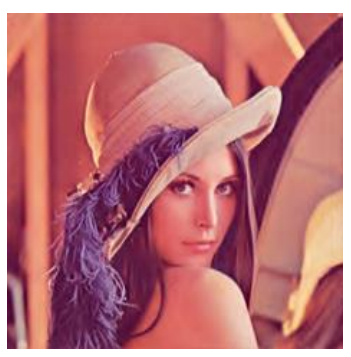

(a)

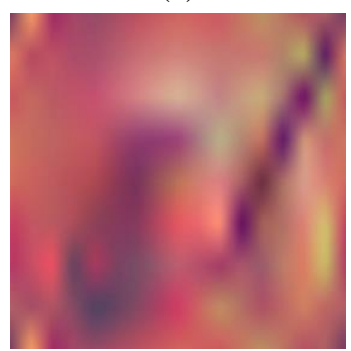

(c)

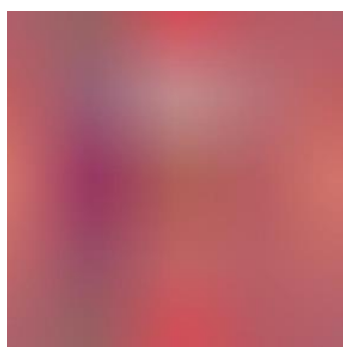

(b)

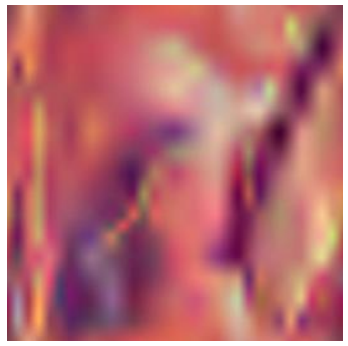

(d)

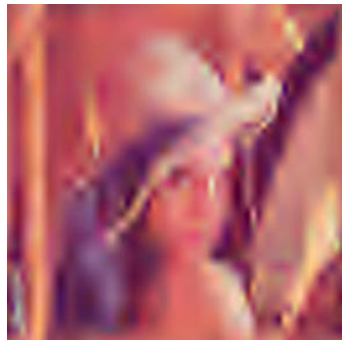

(e)

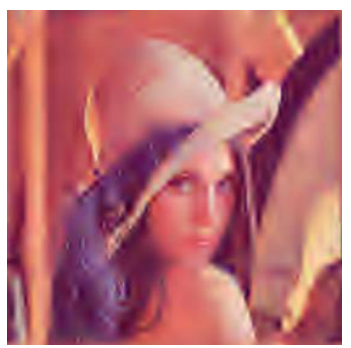

(g)

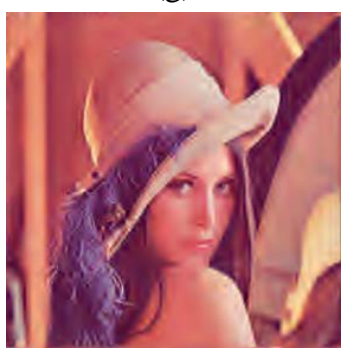

(i)

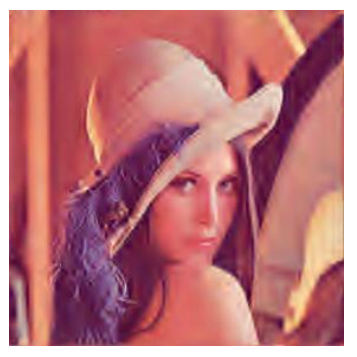

(k)

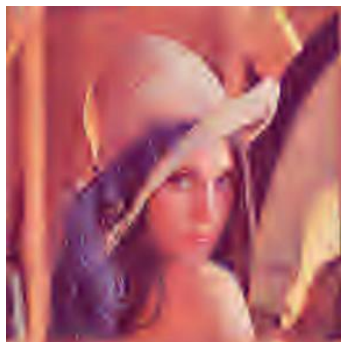

(f)

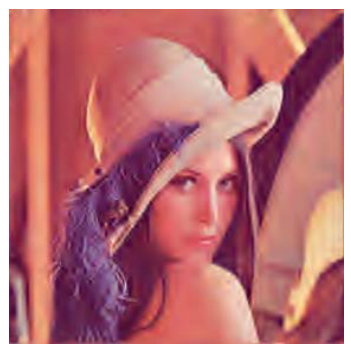

(h)

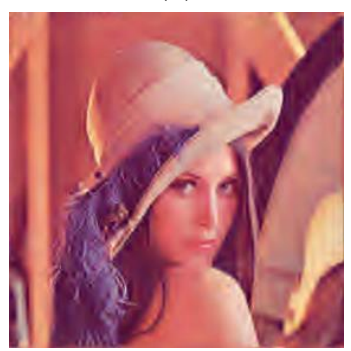

(j)

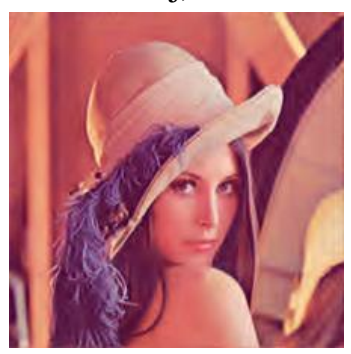

(1)

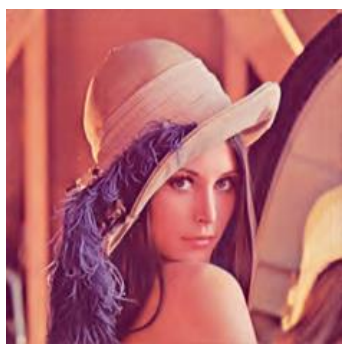

(m)

Fig.9. (a). Original Image (b). Level 1 (c). Level 2 (d). Level 3 (e). Level 4 (f). Level 5 (g). Level 6 (h). Level 7 (i) Level 8 (j) Level 9 (k) Level 10 (l). Level 11 (m) Level 12

From the Fig.9(a) is shown in the original Lena image and Fig.9(b) shown is Filter Bank Level 1, Block Size is 37788 compressed size is 883 , The PSNR value is 22.10 is calculated. The Level 2 Sub Sampling is shown in Fig.9(c). Likewise, this research work is calculated PSNR, MSE and CR for all Sub Levels. The MSE value is gradually decreased for better quality. 
The Fig.9(d) shows the level 3 sub sampling images, IWC uses the DWT Filter Bank methods to reduce the Block Size for visually shows quality image. Fig.9(e) is illustrating RMSE value is 68.52 and CR is 40.26 for the Lena image used in the IWC for Level 4 Sub band.

In Fig.9(f) Sub Band Level 5 indicates the CR 42.16. In Fig.9(g) Sub Band Level 6 specifies the MSE 2814 is gradually decreased for good quality of decomposition. Also, the PSNR value is gradually increased. Fig.9(h) shows the reconstructed images of Sub Band Level 7 image Bit Rate is noted 474.23. Consistently Sub Band Level 8 shown in Fig.9(i).

The Fig.9(j) and Fig.9(k) indicated by Sub Band Level 9 and 10 , respectively. It displays the PSNR value is 36.10 and 37.02 measured with increased compatibility. The IWC methods finding the reduced block size Sub Band level 9 and 10 presents the CR 55.45 and 56.80 respectively

Finally, the Fig.9(l) and Fig.9(m) indicates the Level 11 and 12 presents the CR is 57.12 and 58.20. The Final Sub Level is completely reduced and MSE value is 198.45 to fine tune, the quality of the given original Lena image. The PSNR, MSE, CR, CT and BR parameter are shown in the Section B Table.3.

\subsection{PERFORMANCE ANALYSIS}

The IWC algorithm to observe the variable compression ratio coding arranged the reduced block size. From the results the IWC method can perceive that in case of the reduced compression ratio, the instinctive visual experience of the attain pepper, Mandril and Lena image has to be enhanced the compression ratio and reduced MSE value for better visible effect.

The purpose of selection is common to compare and test IWC algorithms. The desire observations of indexes used in the procedure are signal-to-noise ratio (SNR) and peak signal-tonoise ratio (PSNR). They are computed as follows,

$$
S N R=10 \log \left[\frac{\sum_{m=0}^{M-1} \sum_{n=0}^{N-1}[x(m, n)]^{2}}{\sum_{m=0}^{M-1} \sum_{n=0}^{N-1}[x(m, n)-\hat{x}(m, n)]^{2}}\right]
$$

$$
\begin{gathered}
M S E=\frac{1}{M N} \sum_{m=0}^{M-1} \sum_{n=0}^{N-1}[x(m, n)-\hat{x}(m, n)]^{2} \\
P S N R=10 \log \left[\frac{2^{n} \max -1}{M S E}\right]
\end{gathered}
$$

where $x(m, n)$ and $\hat{x}(m, n)$ are respectively the gray/color value of the original image and reconstructed image on coordinate $(m, n)$. The image size is $M \times N ; n$ max is binary bits used by pixel as to 8bpp.

From Table.1, it is noted with the best compression ratio while the sub band level 12 gets the compression ratio 64.56 and the PSNR value is measured with 40.12. The image quality measurements are calculated in the tested images. The measurements are MSE, RMSE, SNR, CR and BR.

The Table. 2 shows the results of RMSE, SNR, PSNR, CR, BR and CT for Mandrill image. It is observed that the proposed method achieves minimum SNR values is -27.29 and maximum PSNR value is 38.23. At the same time compression ratio is gradually increasing, while the sub band level increases. It is noted that the computation time for the level 12 is 34.84 , whereas the level 1block size gives $3.42 \mathrm{~ms}$.

In Table.3, the results are based on the original Lena uncompressed test image data. Measurement of this table states the quality of the image which calculates peak signal-to-noise ratio, root mean square error, compression ratio, bit rate and computation time measurement for Lena image. It achieves the sub band level 12 PSNR is 39.56 and the Compression ratio is 58.20. After execution of this process, it gives with fastest achievements in $3.48 \mathrm{~ms}$ computation time.

Finally, Table. 4 compares the compression ratio with existing methods. The Fig. 10 shows the comparative performance analysis for Pepper, Lena and Mandrill image and also compares the Compression Ratio with other existing method.

Table.1. Quality Measurement for Pepper

\begin{tabular}{|c|c|c|c|c|c|c|c|c|c|}
\hline Image & Level & MSE & RMSE & SNR & PSNR & Comp size & CR & BR & CT \\
\hline & 1 & 3405.74 & 55.91 & -34.59 & 22.16 & 883 & 9.57 & 2330.17 & 3.42 \\
\cline { 2 - 10 } & 2 & 3667.62 & 50.46 & -32.31 & 24.26 & 1415 & 18.98 & 2097.15 & 7.19 \\
\cline { 2 - 10 } & 3 & 3345.53 & 48.71 & -32.01 & 27.35 & 1875 & 36.45 & 2051.56 & 9.95 \\
\cline { 2 - 10 } & 4 & 1347.39 & 36.45 & -31.17 & 30.15 & 2811 & 42.20 & 1547.08 & 12.97 \\
\cline { 2 - 10 } & 5 & 783.92 & 27.33 & -28.53 & 31.10 & 2918 & 45.26 & 898.78 & 15.81 \\
\cline { 2 - 10 } & 6 & 549.88 & 22.74 & -26.89 & 31.95 & 3397 & 48.23 & 451.54 & 18.86 \\
\cline { 2 - 10 } & 7 & 413.63 & 19.62 & -25.56 & 33.12 & 3645 & 50.51 & 211.36 & 21.92 \\
\cline { 2 - 9 } & 8 & 346.48 & 17.69 & -24.53 & 34.01 & 3880 & 54.30 & 95.13 & 24.88 \\
\cline { 2 - 9 } & 9 & 312.78 & 16.83 & -24.11 & 34.95 & 4032 & 56.12 & 42.67 & 27.88 \\
\cline { 2 - 9 } & 10 & 307.00 & 16.58 & -23.93 & 35.15 & 4141 & 58.25 & 19.61 & 31.56 \\
\cline { 2 - 8 } & 11 & 295.21 & 16.26 & -23.76 & 37.26 & 4202 & 60.51 & 19.01 & 34.03 \\
\cline { 2 - 8 } & 12 & 295.25 & 16.25 & -23.76 & 40.12 & 4263 & 64.56 & 18.38 & 38.48 \\
\hline
\end{tabular}


Table.2. Quality Measurement for Mandrill

\begin{tabular}{|c|c|c|c|c|c|c|c|c|c|}
\hline Image & Level & MSE & RMSE & SNR & PSNR & Comp Size & CR & BR & CT \\
\hline \multirow{5}{*}{} & 1 & 5564.80 & 74.44 & -37.42 & 22.07 & 883 & 4.01 & 2516.58 & 3.42 \\
\cline { 2 - 9 } & 2 & 5564.80 & 74.44 & -37.42 & 23.94 & 883 & 10.25 & 2246.95 & 6.31 \\
\cline { 2 - 9 } & 3 & 5564.80 & 74.44 & -37.42 & 24.16 & 883 & 20.12 & 2169.47 & 9.47 \\
\cline { 2 - 9 } & 4 & 3704.25 & 60.14 & -35.48 & 26.52 & 2571 & 35.23 & 1925.96 & 12.47 \\
\cline { 2 - 9 } & 5 & 2340.42 & 47.86 & -33.50 & 28.61 & 4404 & 39.12 & 1509.95 & 15.56 \\
\cline { 2 - 9 } & 6 & 1627.00 & 40.11 & -32.01 & 30.06 & 3943 & 40.10 & 925.21 & 18.45 \\
\cline { 2 - 9 } & 7 & 1360.44 & 36.59 & -31.19 & 31.45 & 4218 & 45.75 & 512.89 & 21.41 \\
\cline { 2 - 9 } & 8 & 1074.40 & 32.34 & -30.07 & 32.10 & 4758 & 48.23 & 225.50 & 24.56 \\
\cline { 2 - 9 } & 9 & 951.77 & 30.41 & -29.52 & 33.24 & 5046 & 50.12 & 89.28 & 27.81 \\
\cline { 2 - 8 } & 10 & 461.99 & 27.31 & -28.62 & 36.01 & 5824 & 54.24 & 21.22 & 32.19 \\
\cline { 2 - 8 } & 11 & 332.27 & 24.85 & -27.80 & 37.12 & 6298 & 55.12 & 15.12 & 33.67 \\
\cline { 2 - 8 } & 12 & 271.13 & 23.54 & -27.29 & 38.23 & 6548 & 56.10 & 10.10 & 34.84 \\
\hline
\end{tabular}

Table.3. Quality Measurement for Lena

\begin{tabular}{|c|c|c|c|c|c|c|c|c|c|}
\hline Image & Level & MSE & RMSE & SNR & PSNR & Comp Size & CR & BR & CT \\
\hline \multirow{5}{*}{} & 1 & 6604.21 & 78.62 & -37.64 & 22.10 & 883 & 5.42 & 2516.58 & 3.48 \\
\cline { 2 - 10 } & 2 & 6501.21 & 76.63 & -36.14 & 22.85 & 986 & 12.11 & 2246.95 & 6.63 \\
\cline { 2 - 10 } & 3 & 6100.21 & 74.65 & -36.10 & 23.01 & 1045 & 24.62 & 2246.95 & 9.55 \\
\cline { 2 - 10 } & 4 & 5800.21 & 68.52 & -35.94 & 26.45 & 2560 & 40.26 & 2169.47 & 12.55 \\
\cline { 2 - 9 } & 5 & 3980.60 & 62.45 & -35.83 & 28.12 & 3620 & 42.16 & 1731.59 & 15.78 \\
\cline { 2 - 9 } & 6 & 2814.40 & 52.03 & -34.15 & 32.94 & 4173 & 46.30 & 1091.00 & 18.83 \\
\cline { 2 - 9 } & 7 & 1031.80 & 43.36 & -32.40 & 34.62 & 4772 & 49.36 & 474.23 & 21.94 \\
\cline { 2 - 9 } & 8 & 531.82 & 38.72 & -31.32 & 35.16 & 4803 & 54.28 & 204.71 & 24.91 \\
\cline { 2 - 9 } & 9 & 434.66 & 35.63 & -30.48 & 36.10 & 5028 & 55.45 & 86.74 & 28.52 \\
\cline { 2 - 8 } & 10 & 338.64 & 34.32 & -30.13 & 37.02 & 5246 & 56.80 & 34.14 & 29.47 \\
\cline { 2 - 8 } & 11 & 327.40 & 34.18 & -30.10 & 38.15 & 5392 & 57.12 & 14.21 & 30.77 \\
\cline { 2 - 8 } & 12 & 198.45 & 32.52 & -29.67 & 39.56 & 5403 & 58.20 & 12.11 & 32.59 \\
\hline
\end{tabular}

Table.4. Comparison CR with Existing Methods

\begin{tabular}{|c|c|c|c|c|}
\hline Image & SPIHT [20] & JPEG [21] & Daubechies [22] & IWC \\
\hline Pepper & 56.12 & 57.10 & 59.31 & 64.56 \\
\hline Lena & 52.23 & 53.98 & 55.20 & 58.20 \\
\hline Mandrill & 48.24 & 50.12 & 52.45 & 56.10 \\
\hline
\end{tabular}

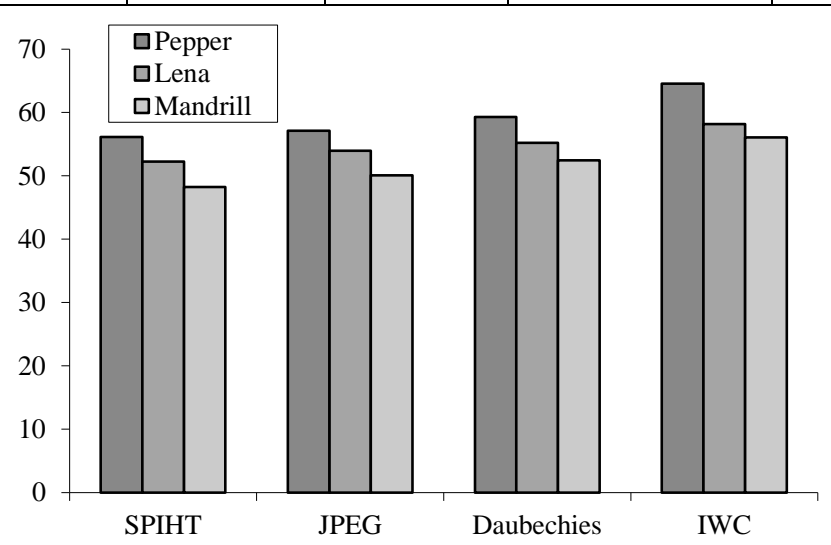

Fig.10. Compare CR with Existing methods

\section{CONCLUSION AND FUTURE WORK}

In this paper explored an Improved Wavelet Compression Algorithm (IWC) produces good quality images for applying both color and grayscale image, this paper proposes the elements into a sequential, vertical and diagonal using filter banks in wavelet domain. It achieves the decrease computation time and increase compression ratio to test with a standard image format. There are many ways to improve the proposed algorithm; the researcher may try to apply the multispectral image, audio, video, photon image. The IWC experimental results show that the method reaches a conclusion of similar or larger to the leading successive presented wavelet algorithm.

\section{REFERENCES}

[1] K. Sayood, "Introduction to Data Compression", Available at: http://rahilshaikh.weebly.com/uploads/1/1/6/3/11635894/da ta_compression.pdf. 
[2] Kamrul Hasan Talukderi and Koichi Harada, "Haar Wavelet Based Approach for Image Compression and Quality Assessment of Compressed Image", International Journal of Applied Mathematics, Vol. 36, pp. 1-9, 2007.

[3] Peter Wayner, "Compression Algorithm for Real Programmer", $1^{\text {st }}$ Edition, Elsevier, 1999.

[4] Markus Kuhn, "Digital Signal Processing", Available at: https://www.cl.cam.ac.uk/teaching/0809/DSP/.

[5] Ken Cabeen and Peter Gent, "Image Compression and the Discrete Cosine Transform", Available at: https://www.math.cuhk.edu.hk/ lmlui/dct.pdf.

[6] Sean Dunn, Available at: http://davis.wpi.edu/ matt/courses/color

[7] Colm Mulcahy, "Image Compression using the HAAR Wavelet Transform", Spelman Science and Math Journal, pp. 22-31, 1997.

[8] Ryuji Matsuoka, Mitsuo Sone, Kiyonari Fukue, Kohei Cho and Haruhisa Shimoda, "Quantitative Analysis of Image Quality of Lossy Compression Images", Available at: https://pdfs.semanticscholar.org/e929/fe4e037d80a2265490 54fd35bced632e2009.pdf.

[9] James Z. Wang, "Wavelets and Imaging Informatics: A Review of the Literature", Journal of Biomedical Informatics, Vol. 34, No. 2, pp. 129-141, 2001.

[10] Yves Meyer, "Wavelets Algorithms and Applications", SIAM Journal on Applied Mathematics, Vol. 53, No. 1, pp. 1-6, 1993.

[11] Charles K. Chui, "An Introduction to Wavelets", Academic Press, 1992.

[12] C.K. Chui, "Wavelets: A Tutorial in Theory and Applications", Academic Press, 1992.

[13] Subhasis Saha, "Image Compression - from DCT to Wavelets: A Review", Crossroads, Vol. 6, No. 3, pp. 12-21, 2000.

[14] Li Wern Chew, Li-Minn Ang and Kah Phooi Seng, "Survey of Image Compression Algorithms in Wireless Sensor Networks", Proceedings of International Symposium on Information Technology, pp. 1-9, 2008.
[15] D. Cruz, T. Ebrahimi, J. Askelof, M. Larsson and C. Christopoulos, "Coding of Still Picture", Proceedings of $45^{\text {th }}$ SPIE Applications of Digital Image Processing, Vol. 4115, pp. 1-10, 2000.

[16] Suchitra Shrestha and Khan Wahid, Hybrid DWT-DCT Algorithm for Biomedical Image and Video Compression Applications, Proceedings of $10^{\text {th }}$ IEEE International Conference on Information Sciences, Signal Processing and their Applications, pp. 280-283, 2010.

[17] Swapna Subudhiray and Abhishek Kr. Srivastav, "Implementation of Hybrid Dwt-Dct Algorithm For Image Compression: A Review", International Journal of Research in Engineering and Applied Sciences, Vol. 2, No. 2, pp. 1200-1210, 2012.

[18] M. Shwetha, P. Ashwini and B.M. Sujatha, "Analysis of Image Compression Algorithms in WSN: A Review", International Journal of Science, Engineering and Technology Research, Vol. 3, No. 4, pp. 1029-1032, 2014.

[19] Sonja Grgic, Kresimir Kers and Mislav Grgic, "Image Compression using Wavelets", Proceedings of IEEE International Symposium on Industrial Electronics, pp. 99 104, 1999.

[20] R.Sudhakar, R Karthiga and S. Jayaraman, "Image Compression using Coding of Wavelet Coefficients-A Survey", ICGST-GVIP Journal, Vol. 5, No. 6, pp. 25-38, 2005.

[21] Renu Sharma and Matish Garg, "Comparative analysis of JPEG DCT, Haar and Daubechies Wavelet, Fractal for Image Compression", International Journal of Advanced Research in Computer Science and Software Engineering, Vol. 4, No. 1, pp. 1227-1234, 2014.

[22] S. Sridhar, P. Rajesh Kumar and K.V. Ramanaiah, "Wavelet Transform Techniques for Image Compression-An Evaluation", International Journal of Image, Graphics and Signal Processing, Vol. 2, pp. 54-67, 2014.

[23] USC-SIPI Image Database, Available at: http://sipi.usc.edu/database/database.php?volume=misc, Accessed on 2014. 